University of Nebraska - Lincoln

DigitalCommons@University of Nebraska - Lincoln

1987

\title{
Differential Cross Sections for Ionization of Water Vapor by High- Velocity Bare Ions and Electrons
}

\author{
J. H. Miller \\ Radiological Physics Section, Pacific Northwest Laboratory, Richland, Washington \\ W. E. Wilson \\ Radiological Physics Section, Pacific Northwest Laboratory, Richland, Washington
}

S. T. Manson

Georgia State University, Atlanta, Georgia

M. Eugene Rudd

University of Nebraska - Lincoln, erudd@unl.edu

Follow this and additional works at: https://digitalcommons.unl.edu/physicsrudd

Part of the Physics Commons

Miller, J. H.; Wilson, W. E.; Manson, S. T.; and Rudd, M. Eugene, "Differential Cross Sections for Ionization of Water Vapor by High-Velocity Bare Ions and Electrons" (1987). M. Eugene Rudd Publications. 22.

https://digitalcommons.unl.edu/physicsrudd/22

This Article is brought to you for free and open access by the Research Papers in Physics and Astronomy at DigitalCommons@University of Nebraska - Lincoln. It has been accepted for inclusion in M. Eugene Rudd Publications by an authorized administrator of DigitalCommons@University of Nebraska - Lincoln. 


\title{
Differential cross sections for ionization of water vapor by high-velocity bare ions and electrons
}

\author{
Journal of Chemical Physics 86, 157 (1987); DOI:10.1063/1.452774
}

Issue Date: 1 January 1987

J. H. Miller and W. E. Wilson

Radiological Physics Section, Pacific Northwest Laboratory, Richland, Washington 99352

S. T. Manson

Department of Physics and Astronomy, Georgia State University, Atlanta, Georgia 30303

M. E. Rudd

Department of Physics, University of Nebraska-Lincoln, Lincoln, Nebraska 68588-0111

\begin{abstract}
A semiempirical model of single differential cross sections (SDCS) for ionization of water vapor by fast electrons and bare ions is presented. At low secondary-electron energy, the model is based on an asymptotic expansion of the first Born approximation with coefficients, that are independent of projectile properties, evaluated from experimental photoabsorption and protonimpact ionization data. As the secondary-electron energy increases, the model converges to a binary-encounter approximation. Comparisons with measured differential, total, and dissociative cross sections for ionization of water by fast electrons are used to test the model. For primary electrons with energy greater than about $500 \mathrm{eV}$, agreement with these data is generally within experimental uncertainty; however, some discrepancies of uncertain origin exist.
\end{abstract}

The Journal of Chemical Physics is copyrighted by The American Institute of Physics.

History: Received 8 August 1986; accepted 18 September 1986

Permalink: http://link.aip.org/link/?JCPSA6/86/157/1 


\title{
Differential cross sections for ionization of water vapor by high-velocity bare ions and electrons
}

\author{
J. H. Miller and W. E. Wilson \\ Radiological Physics Section, Pacific Northwest Laboratory, Richland, Washington 99352
}

S. T. Manson

Department of Physics and Astronomy, Georgia State University, Atlanta, Georgia 30303

M. E. Rudd

Department of Physics, University of Nebraska-Lincoln, Lincoln, Nebraska 68588-0111

(Received 8 August 1986; accepted 18 September 1986)

\begin{abstract}
A semiempirical model of single differential cross sections (SDCS) for ionization of water vapor by fast electrons and bare ions is presented. At low secondary-electron energy, the model is based on an asymptotic expansion of the first Born approximation with coefficients, that are independent of projectile properties, evaluated from experimental photoabsorption and protonimpact ionization data. As the secondary-electron energy increases, the model converges to a binary-encounter approximation. Comparisons with measured differential, total, and dissociative cross sections for ionization of water by fast electrons are used to test the model. For primary electrons with energy greater than about $500 \mathrm{eV}$, agreement with these data is generally within experimental uncertainty; however, some discrepancies of uncertain origin exist.
\end{abstract}

\section{INTRODUCTION}

Semiempirical models based on Bethe's ${ }^{1}$ theory of energy loss by fast charged particles have been extremely useful in extending the data base of differential ionization cross sections. $^{2-6}$ This approach requires evaluation of coefficients in an asymptotic expansion of the first Born approximation. ${ }^{7}$ Coefficients of the lowest-order term in this expansion are related to the target's optical oscillator strength, which can be deduced from photoabsorption data. ${ }^{8}$ Although the optical oscillator strength is the dominant factor determining the spectrum of low-energy secondary electrons ejected by highvelocity ions, higher-order terms can also be important. We have used proton-impact-ionization data in conjunction with photoionization cross sections to evaluate coefficients of the first two terms in the Bethe expansion. ${ }^{4,5}$ Like the optical oscillator strengths, the higher-order coefficients are also independent of projectile properties. Hence, coefficients that are based on data obtained with high-energy protons can be used to predict single differential cross sections (SDCS) for ionization of the target by any bare ion or electron of sufficient energy to make the Bethe theory valid. ${ }^{6}$

The accuracy of this data extrapolation technique is mainly limited by experimental uncertainty in the photoionization data and proton-impact SDCS used to determine the expansion coefficients. Experimental uncertainty is particularly large in measurements of SDCS for ejection of lowenergy secondary electrons from water by proton impact. ${ }^{9}$ Since total cross sections for ionization by charged particles are determined mainly by the ejection of low energy secondary electrons, we use recent measurements of total cross sections for ejection of electrons from water by protons ${ }^{10}$ to augment the differential ionization data in evaluating the higher-order coefficients in our model. This approach is facilitated by the use of a simple analytic function for the dependence of the higher-order coefficients on secondary-elec- tron energy. For this purpose, we have adapted a function employed by Dillon and Inokuti ${ }^{11-13}$ and by Dillon et al.$^{14}$ to fit optical oscillator strengths, generalized oscillator strengths (GOS), and secondary-electron spectra.

In the following section of this paper, the theoretical basis of our semiempirical model is briefly reviewed. The next section describes application of the model to water vapor and compares calculated differential, total, and dissociative cross sections for ionization of $\mathrm{H}_{2} \mathrm{O}$ by fast electrons with experimental data. ${ }^{15-17}$ Our results are summarized in the final section.

\section{THEORY}

For a target that contains $N$ subshells with ionization potentials $I_{k}$, the first Born approximation to the cross section for ejection of secondary electrons with energy between $W$ and $W+d W$ by an ion with charge $Z$, mass $M$, and velocity $v$ can be written in the form

$$
\frac{d \sigma}{d W}=\frac{4 \pi a_{0}^{2} Z^{2}}{T} \sum_{k=1}^{N} \int_{Q_{\min }}^{Q_{\max }} \frac{d Q}{Q} \frac{R^{2}}{E_{k}} \frac{d f_{k}(Q)}{d W},
$$

where $a_{0}$ is the Bohr radius (0.529 $\AA$ ), $R$ is the Rydberg $(13.6 \mathrm{eV})$, and $T=\frac{1}{2} m v^{2}$ with $m$ the electron mass. $E_{k}=I_{k}+W$ is the energy transfer in the collision, $d f_{k}(Q) / d W$ is the GOS of the target, and $Q=\left(K a_{0}\right)^{2}$ where $\hbar K$ is the momentum transfer. The limits on $Q$, which are determined by conservation of energy and momentum, can be approximated by

$$
Q_{\min }=\frac{1}{4} \frac{E_{k}^{2}}{R T}\left(1+\frac{m E_{k}}{2 M T}\right)
$$

and

$$
Q_{\max }=\frac{4 T}{R}\left(\frac{M}{m}\right)^{2}\left(1-\frac{m E_{k}}{2 M T}\right),
$$


where terms of order $\left(m E_{k} / M T\right)^{2}$ and higher have been neglected. ${ }^{7}$

Calculation of the GOS requires accurate wave functions for both the bound and continuum states of the target, which are not currently available for most molecular targets. Hence, approximations to the result given in Eq. (1) are needed. Once such approximation was derived by Bethe, ${ }^{1}$ who recognized that the dipole part of the Coulomb interaction predominates in collisions with small momentum transfer. Hence, cross sections for small energy loss by fast charged particles are closely related to photoabsorption by the target. This relationship is seen more clearly by using the methods discussed by Inokuti ${ }^{7}$ to rewrite Eq. (1) in the form

$$
\begin{aligned}
\frac{d \sigma}{d W}= & \frac{4 \pi a_{0}^{2} Z^{2}}{T} \sum_{k=1}^{N} \frac{R^{2}}{E_{k}} \frac{d f_{K}}{d W} \ln \left(\frac{4 R T}{E_{k}^{2}}\right) \\
& +b_{k}(W)+O\left(\frac{E_{k}}{T}\right),
\end{aligned}
$$

where

$$
\begin{aligned}
b_{k}(W)= & \int_{0}^{1} \frac{d Q}{Q} \frac{R^{2}}{E_{k}}\left[\frac{d f_{k}(Q)}{d W}-\frac{d f_{k}}{d W}\right] \\
& +\int_{1}^{\infty} \frac{d Q}{Q} \frac{d f_{k}(Q)}{d W},
\end{aligned}
$$

$d f_{k} / d W$ is the partial optical oscillator strength for ejection of an electron with energy $W$ by photoionization of the $k^{\text {th }}$ subshell of the target, and $O\left(E_{k} / T\right)$ denotes terms in the Bethe expansion that are of order $E_{k} / T$ and higher.

Since $b_{k}(W)$ is independent of projectile properties, SDCS data at a single ion energy can in principle be use to evaluate

$$
b(W)=\sum_{k=1}^{N} b_{k}(W)
$$

by the equation

$$
\begin{aligned}
b_{\exp }(W)= & \left.\frac{T}{4 \pi a_{0}^{2} Z^{2}} \frac{d \sigma}{d W}\right|_{\exp } \\
& -\sum_{k=1}^{N} \frac{R^{2}}{E_{k}} \frac{d f_{k}}{d W} \ln \left(\frac{4 R T}{E_{k}^{2}}\right)
\end{aligned}
$$

when the secondary-electron energy is sufficiently low to neglect the terms of order $E_{k} / T$. These empirical coefficients can then be used to predict the cross sections for ejection of low-energy electrons by bare ions and electrons at any energy for which the Bethe theory is valid. We have found this to be an efficient and accurate method for extrapolating differential ionization data. ${ }^{4-6}$

The results of this procedure are, of course, very sensitive to experimental error in the differential ionization data used in Eq. (6). The effect of random error can be reduced by averaging results obtained for $b_{\exp }(W)$ with SDCS data at several proton energies; however, systematic errors may still be significant. Hence, it is desirable to have other types of experimental data to aid in determination of the model coefficients. Total ionization cross sections are most helpful in this regard since they are sensitive to the low-energy part of the secondary-electron spectrum. Stopping power data is also useful, but less sensitive to the details of the spectrum at low secondary-electron energy.

Use of moments of the secondary-electron spectrum to improve estimates of the Bethe coefficients is facilitated by two analytic properties of $b(W)$. First, an approximate highenergy limit for $b(W)$ can be calculated from the behavior of the GOS at large energy transfer, where values of $Q$ in the neighborhood of $E_{k} / R$ predominate in Eq. (5). In this region, a binary encounter approximation (BEA) to the GOS is adequate, ${ }^{18}$ and by following the algebra outlined by Inokuti ${ }^{7}$ one can show that $b(W)$ is approximately equal to

$$
b_{0}(W)=\sum_{k=1}^{N} n_{k} R^{2}\left(1+\frac{4 U_{k}}{3 E_{k}}\right) / E_{k}^{2},
$$

where $n_{k}$ is the number of electrons in the $k$ th subshell and $U_{k}$ is their average kinetic energy. If Bethe coefficients deduced from experimental data converge to this semiclassical limit, then we can make a smooth transition between the Bethe-Born approximation at low secondary-electron energy and the BEA at large $W$. A model of SDCS over the whole range of secondary-electron energies is essential for accurate evaluation of moments of the spectrum.

The second analytic property of $b(W)$ that is useful in evaluation of total cross sections and stopping power is the fact that the ratio $b / b_{0}$ is well represented by a power series in the variable $W /\left(W+I_{1}\right)$, where $I_{1}$ is the lowest ionization potential of the target. ${ }^{13}$ This provides a simple functional form for the dependence of higher-order coefficients on secondary-electron energy. The parameters of this function can be varied to simultaneously optimize agreement with SDCS, total ionization cross sections, and stopping power.

\section{RESULTS AND DISCUSSION}

Bethe coefficients deduced from SDCS for ionization of water vapor by high-energy protons ${ }^{9}$ and electrons ${ }^{15}$ are shown in Figs. 1 (A) and 1(B). The solid curve shows the fit to only the proton-impact data (open symbols) obtained with the function

$$
b(W)=b_{0}(W) \sum_{i=0}^{3} c_{i} x^{i},
$$

where $b_{0}(W)$ is given by Eq. (7) with $U_{k}=g I_{k}$ and $X=W /\left(W+I_{1}\right)$. The constraint

$$
\sum_{i=0}^{3} c_{i}=1
$$

is placed on the fitting parameters so that the functional representation of $b(W)$ converges to the semiclassical limit as $W$ increases. This limit is shown by the dashed line in Fig. 1 (B). The error bars indicate the uncertainty in $b_{\exp }(W)$ that results from a $\pm 20 \%$ uncertainty in the proton-impact SDCS. Larger uncertainty is expected for secondary-electron energies below $10 \mathrm{eV}$. The fitting parameters $c_{1}, c_{2}, c_{3}$, and $g$ were also optimized for agreement with total cross sections for ejection of electrons from water by protons with energy between 0.5 and $5 \mathrm{MeV}^{10}$ and with the contribution of ionization to the stopping power of protons in water vapor. These results are shown in Fig. 2. Values of the adjusta- 

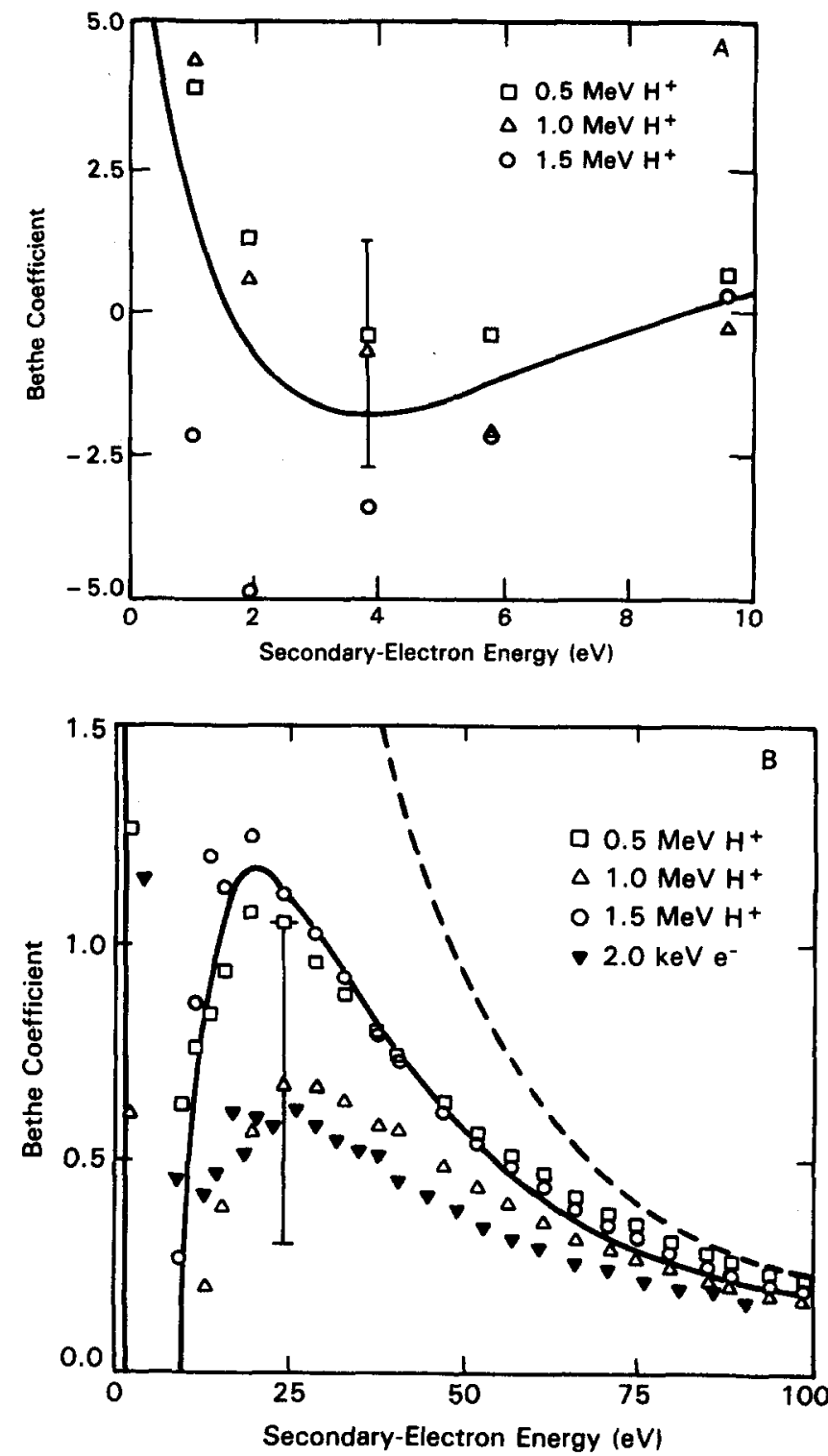

FIG. 1. Bethe coefficients at low (A) and intermediate (B) secondary-electron energies deduced from photoionization data (Refs. 8 and 22-24) and single differential cross sections (SDCS) for ionization of water vapor by high-energy electrons (Ref. 15) and protons (Ref.9). Solid curve shows the analytic representation of Bethe coefficients optimized for agreement with proton-impact ionization data (Refs. 9, 10, and 19). Dashed curve is the result obtained from a binary-encounter approximation (Ref. 18).

ble parameters that give the best fit to the data are $c_{1}=-2.16, c_{2}=5.00, c_{3}=-2.02$, and $g=5.36$. The optimum value of the parameter $g$ is consistent with $a b$ initio calculations for the outermost subshell of the water molecule [Stevens (private communications); see Ref. 15].

The ionization component of stopping power was obtained by subtracting the contribution of discrete excitations from the total stopping power calculated by the Bethe formula with a mean excitation energy of $71.6 \mathrm{eV} .{ }^{19}$ Since the contribution to the stopping power from excitation of discrete levels is small for proton energies above $0.5 \mathrm{MeV},{ }^{20}$ the uncertainty of these estimates of the contribution to stopping power from ionizing collisions is about $5 \%$. Calculation of stopping power by integration of secondary-electron spectra requires SDCS for ionization of individual subshells of the

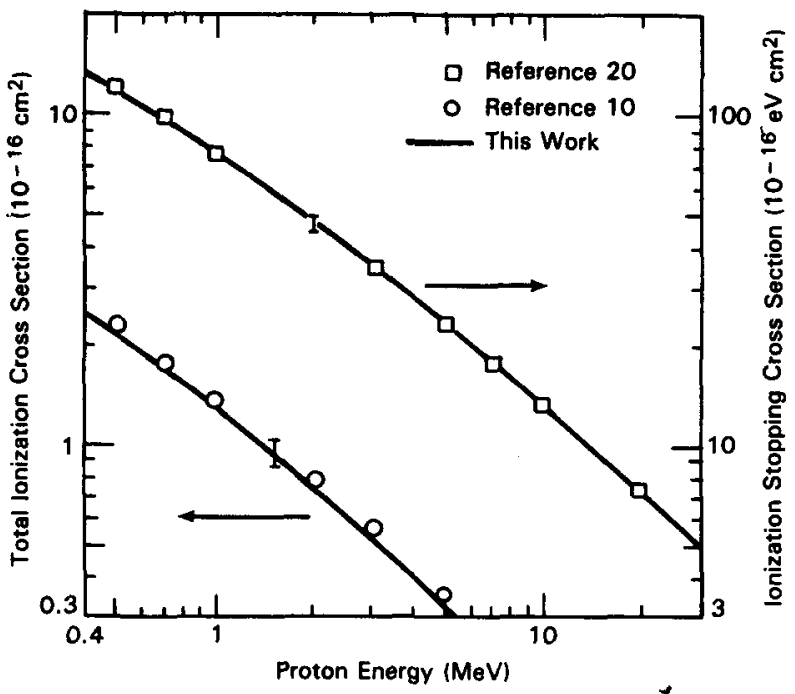

FIG. 2. Comparison of model calculations with measured total cross sections for ionization of water vapor by protons and the contribution of ionization to the stopping power of protons in water.

target. Hence, we have made the additional assumption that the ratio $b / b_{0}$ is independent of subshell. This assumption is not needed for calculations of total ionization cross sections. In Fig. 2, one can see that although the calculations of total ionization cross sections with optimum choice of the adjustable parameters are within experimental uncertainty, calculated values are slightly less than experiment. ${ }^{10}$ Hence, these experimental data are most consistent with the larger values of $b_{\exp }(W)$ for secondary-electron energies below $50 \mathrm{eV}$.

Figure 3 compares predictions of our model with experimental SDCS for ionization of water vapor by 0.5 and $2 \mathrm{keV}$

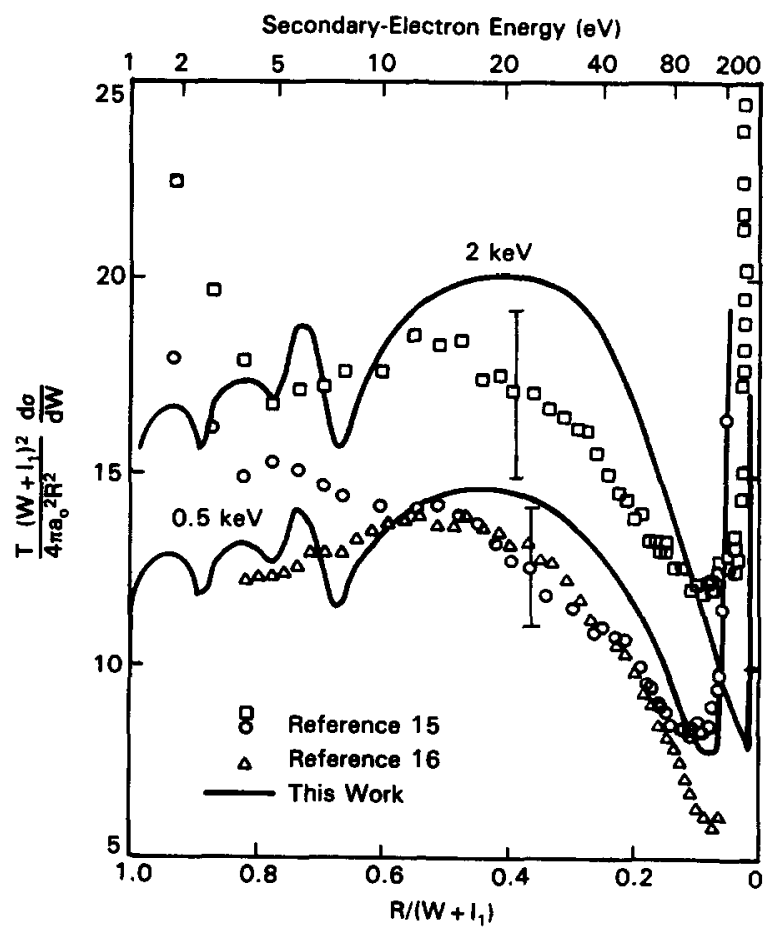

FIG. 3. Comparison of calculated secondary-electron spectra with experimental data on ionization of water vapor by 0.5 and $2.0 \mathrm{keV}$ electrons. $R$ denotes the Rydberg energy ( $13.6 \mathrm{eV}), a_{0}$ is the Bohr radius $(0.529 \AA)$, and $I_{1}$ is the lowest ionization potential of water vapor $(12.6 \mathrm{eV}) . T$ and $W$ denote the primary and secondary electron energy, respectively. 
electrons. ${ }^{15,16}$ In these calculations, $b(W)$ was obtained by multiplying the polynomial representation of $b / b_{0}$ that gave optimum agreement with the proton-impact ionization data by a BEA that includes electron exchange. ${ }^{18}$ The structures that are present in the calculations at low secondary-electron energy result from structure in the photoabsorption data for the three lowest ionization continuua of $\mathrm{H}_{2} \mathrm{O}$, as discussed by Tuckwell and $\mathrm{Kim}^{21}$ for the $\mathrm{N}_{2}$ molecule. Their position and magnitude are very sensitive to the uncertainty in partial optical oscillator strengths near threshold. ${ }^{22-24}$ Vibrational states that are neglected in our model probably tend to deemphasize the importance of these structures in the secondaryelectron spectrum. The error bars illustrate an experimental uncertainty of $\pm 12 \%$. Measurements at secondary-electron energies less than $15 \mathrm{eV}$ have larger uncertainty that approaches $\pm 50 \%$ for $2 \mathrm{eV}$ secondary electrons. ${ }^{15}$

The broad structure in our calculations that has a maximum for secondary-electron energies near $20 \mathrm{eV}$ results from a maximum of $b(W)$ in this energy range. From the results shown in Fig. 1(B), it is clear that better agreement with electron-impact SDCS could be achieved by using Bethe coefficients deduced from $1 \mathrm{MeV}$ proton-impact SDCS rather than the $b(W)$ that optimizes agreement with all of the available proton-impact ionization data. Systematic variations in $b_{\exp }(W)$ with proton energy could result from our neglect of terms of order $E_{k} / T$ in the Bethe expansion or from ionization mechanisms that are not included in the first Born approximation, e.g., charge transfer to continuum states. ${ }^{25}$ However, since these effects decrease with increasing proton energy, ${ }^{26} b_{\exp }(W)$ deduced from the highest-energy-proton data should give the best agreement with the electron-impact SDCS. We do not observe this to be the case and therefore tend to attribute the discrepancy between our model calculations and the electron-impact SDCS data for secondary electrons with energy near $25 \mathrm{eV}$ to systematic experimental error in measurements of secondary electron spectra. This discrepancy could also be related to the BEA component of our model in the following way. Although we observe convergence of the experimentally deduced Bethe coefficients to results expected from a BEA as the secondary-electron energy approaches $100 \mathrm{eV}$ [see Fig. 1(B)], the binary-encounter theory for proton-impact ionization systematically underestimates the measured SDCS at large secondary-electron energies by $10 \%$ to $15 \%$. Hence, the larger values of $b(W)$ for small $W$ that give the best agreement with total ionization cross sections and stopping power may be compensating for a deficiency in the BEA for calculating proton-impact SDCS at large secondary-electron energies.

As the primary-electron energy increases, a significant discrepancy between our model and the electron-impact data develops near the minimum in the cross section (i.e., when the two electrons in the final state have nearly equal energy). This can be seen more clearly in Fig. 4, which compares our calculations with the electron-impact differential ionization data on a plot that emphasizes the large-energytransfer part of the secondary-electron spectrum. This discrepancy between the predictions of our model and the experimental electron-impact SDCS is somewhat dependent on how electron exchange is treated in the $\mathrm{BEA}^{18}$; however,

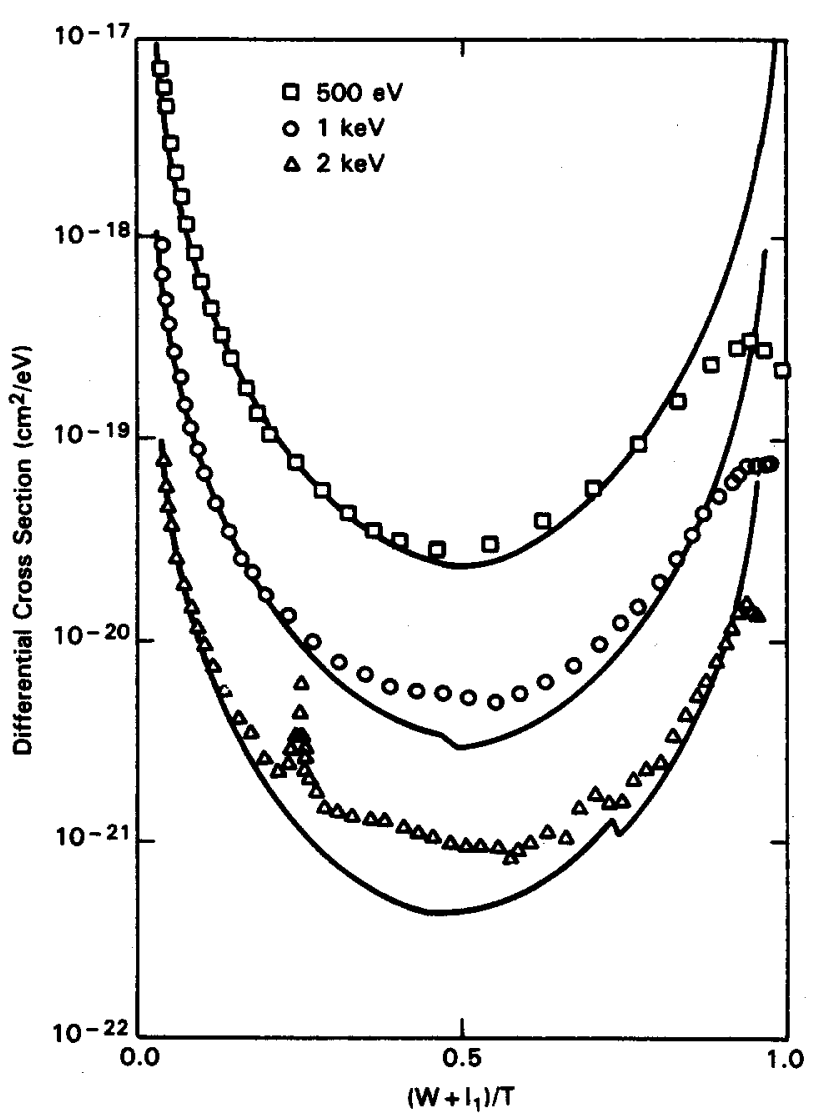

FIG. 4. Comparison of theoretical and experimental single differential cross sections (SDCS) for ionization of water vapor by electron impact (Ref. 15) on a plot that emphasizes results at large energy transfer. $T, W$, and $I_{1}$ have the same meaning as in Fig. 3.

it may also be due to difficulties in measuring the rather small cross sections in this energy region. Preliminary experiments suggest that a more complete suppression of spurious electrons will further decrease the measured cross sections in the neighborhood of their minimum value.

The peak in the experimental $2 \mathrm{keV}$ electron-impact data for secondary electrons with energy near $500 \mathrm{eV}$ is due to Auger electrons that are not included in our model. The sharp discontinuities in the calculated results are due to turning off individual subshells when ejection of a secondary electron with energy $W$ results in an energy loss that is greater than the primary-electron energy.

Figure 5 compares calculated total cross sections for ionization of water by electron impact with the data of Schutten et al. ${ }^{17}$ These data were not used in the optimization procedure, and the fact that our calculations are systematically larger than Schutten's data reflects the influence of the more recent proton-impact total cross section measurements ${ }^{10}$ on our model. Nevertheless, for electron energies above $0.4 \mathrm{keV}$, the agreement is still within the estimated experimental error of $15 \%$. The systematic divergence of the calculated total cross sections from the experimental data for electrons with energy less than $0.4 \mathrm{keV}$ is probably a good indicator of the limit of applicability of our model as the velocity of the projectile decreases.

Incorporation of the optical oscillator strengths for fragmentation of $\mathrm{H}_{2} \mathrm{O}$ by photoionization ${ }^{22}$ allows us to 


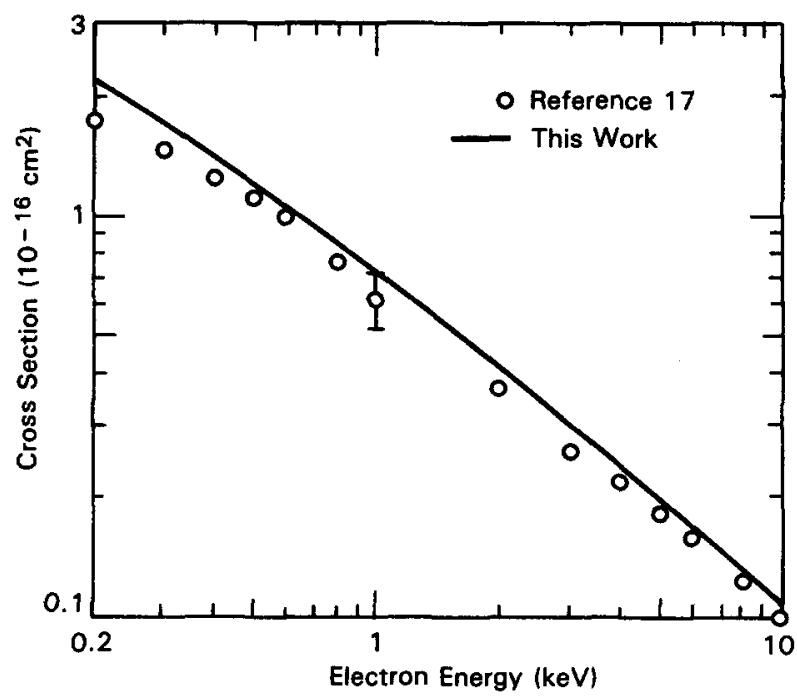

FIG. 5. Comparison of theoretical total cross sections with experimental data (Ref. 17) on ionization of water vapor by electron impact.

compare our model with Schutten's dissociative ionization cross sections. Branching ratios for the production of $\mathrm{H}_{2} \mathrm{O}^{+}, \mathrm{OH}^{+}, \mathrm{H}^{+}$, and $\mathrm{O}^{+}$that are approximately independent of electron energy above $0.5 \mathrm{keV}$, are compared in Table I. Our result for the $\mathrm{H}^{+}$channel is only half as great as the result for the $\mathrm{OH}^{+}$channel in contrast to Schutten's data, which gives a branching ratio of $\mathrm{H}^{+}$that is somewhat larger than the branching ratio for $\mathrm{OH}^{+}$. Our result is a consequence of the small oscillator strengths for production of $\mathrm{H}^{+}$when the energy transfer is less than $30 \mathrm{eV}$. In this region of energy transfer, oscillator strengths for production of $\mathrm{H}^{+}$are about $30 \%$ of the oscillator strengths for production of $\mathrm{OH}^{+}$. Preliminary results obtained with high-energy protons tend to support the model predictions (DuBois, private communication ).

\section{SUMMARY AND CONCLUSIONS}

We have developed a semiempirical model for calculating cross sections, differential in the energy of secondary electrons, for ionization of atomic and molecular targets by high-velocity bare ions and electrons. In this paper, we reexamine the application of the model to water vapor in light of new total ${ }^{10}$ and differential ${ }^{15}$ cross section measurements. The model is based on evaluation of coefficients, which are independent of the projectile, in Bethe's asymptotic expansion of the first Born approximation ${ }^{1,7}$ through the use of photoabsorption $^{8,22-24}$ and proton-impact ${ }^{9,10}$ ionization data. An analytic function suggested by Dillon and Inokuti ${ }^{11}$ is used to represent the dependence of the Bethe coefficients

TABLE I. Branching ratios for dissociative ionization of water vapor.

\begin{tabular}{lcccc}
\hline \hline & $\mathrm{H}_{2} \mathrm{O}^{+}$ & $\mathrm{OH}^{+}$ & $\mathrm{H}^{+}$ & $\mathrm{O}^{+}$ \\
\hline Theory & 0.72 & 0.16 & 0.10 & 0.02 \\
Expt. $^{\mathrm{a}}$ & $\mathbf{0 . 6 2}$ & 0.16 & 0.20 & 0.02 \\
\hline \hline
\end{tabular}

"Schutten et al., 1966. on secondary-electron energy. This allows us to optimize agreement with total ionization cross sections and stopping power data, while fitting the Bethe coefficients deduced from differential ionization data. This is particularly important for water vapor since experimental difficulties enhance the uncertainty of SDCS at low secondary-electron energies. However, this approach makes the optimum $b(W)$ somewhat dependent on approximations used to calculate SDCS for ejection of high-energy secondary electrons. In this region of the spectrum, we have used a BEA, ${ }^{18}$ which gives results slightly less than the measured proton-impact SDCS, but nevertheless agrees with these data to within the estimates of experimental uncertainty.

The analytic representation of Bethe coefficients that gave the best agreement with the available data on ionization of water by high-energy protons was then used to predict SDCS ionization of water by fast electrons ${ }^{15,16}$ (as well as total and dissociative ionization cross sections ${ }^{17}$ ). Generally, for primary electrons with $500 \mathrm{eV}$ or greater energy, agreement between model calculations and experimental data is within estimates of experimental uncertainty; however, some significant differences were found. For secondary electrons with energy between 25 and $50 \mathrm{eV}$, the model predicts SDCS that are slightly larger than the experimental data. ${ }^{15,16}$ As the primary-electron energy increases, the model predicts results that are considerably smaller than experimental SDCS ${ }^{15}$ when the two electrons in the final state have approximately equal energy. Finally, the model predicts a branching ratio for $\mathrm{H}^{+}$production that is about half that observed experimentally. ${ }^{17}$ The sources of these discrepancies are unknown at present and deserve further theoretical and experimental investigation. In addition to providing a means for extending the data base of differential ionization cross sections, our model also tests the internal consistency of related ionization measurements (i.e., SDCS, total ionization cross sections, stopping power, etc.). Our analysis does not reveal any major inconsistencies among these data for water vapor.

\section{ACKNOWLEDGMENTS}

The authors gratefully acknowledge helpful discussions with Dr. Mitio Inokuti, Dr. Yong-Ki Kim, and Dr. Herwig Paretzke. This work was supported by the U.S. Department of Energy under Contract No. DE-ACO6-76RLO 1980, U.S. Army Research Office under Contract No. DAAG-29-83K-0054, and the National Science Foundation under Contract No. PHY-8401328.

'H. Bethe, Ann. Phys. 5, 325 (1930).

${ }^{2}$ Y.-K. Kim, Radiat. Res. 61, 21 (1975); 64, 96, 205 (1975).

${ }^{3}$ H. G. Paretzke and M. J. Berger, in Microdosimetry, Proceedings of the Sixth Symposium, edited by J. Booz and H. G. Ebert (Harwood, London, 1978), p. 749.

${ }^{4} J$. H. Miller L. H. Toburen and S. T. Manson Phys. Rev. A 27, 1337 (1983).

${ }^{3}$ W. E. Wilson, J. H. Miller, L. H. Toburen, and S. T. Manson, J. Chem. Phys. 80, 5631 (1984).

${ }^{6}$ J. H. Miller and S. T. Manson, Phys. Rev. A 29, 2435 (1984).

${ }^{7}$ M. Inokuti, Rev. Mod. Phys. 43, 297 (1971). 
${ }^{8}$ J. Berkowitz, Photoabsorption, Photoionization and Photoelectron Spectroscopy (Academic, New York, 1979).

${ }^{9}$ L. H. Toburen and W. E. Wilson, J. Chem. Phys. 66, 5202 (1977)

${ }^{10}$ M. E. Rudd, T. V. Goffe, R. D. DuBois, and L. H. Toburen, Phys. Rev. A 31, 492 (1985).

${ }^{11} M$. A. Dillon and M. Inokuti, J. Chem. Phys. 74, 6271 (1981).

${ }^{12}$ M. A. Dillon and M. Inokuti, J. Chem. Phys. 82, 4415 (1985)

${ }^{13}$ M. Inokuti and M. A. Dillon, in Physics of Ionized Gases, Proceedings of the XIIth Yugoslav Summer School and International Symposium, September 1984, Sibenik, Yugoslavia (in press).

${ }^{14}$ M. A. Dillon, M. Inokuti, and Z. -W, Wang, Radiat. Res. 102, 151 (1985).

${ }^{15}$ M. A. Bolorizadeh and M. E. Rudd, Phys. Rev. A 33, 882 (1986)

${ }^{16}$ C. B. Opal, E. C. Beaty, and W. K. Peterson, At. Data Nucl. Data Tables 4, 209 (1972).

${ }^{17}$ J. Schutten, F. J. de Heer, H. R. Moustafa, A. J. H. Boerboom, and J. Kistemaker, J. Chem. Phys. 44, 3924 (1966).

${ }^{18} \mathrm{~L}$. Vriens, in Case Studies in Atomic Collision Physics I, edited by E. W. McDaniel and M. R. C. McDowell (North-Holland, Amsterdam, 1969), p. 335.

${ }^{19}$ International Commission on Radiation Units and Measurements, Stopping Powers for Electrons and Positrons, Report 37 Bethesda, MD, 1984, p. 25.

${ }^{20}$ W. E. Wilson, L. H. Toburen, J. H. Miller, and R. D. DuBois, in Proceedings, Workshop on Electronic and Ionic Collision Cross Sections Needed in the Modeling of Radiation Interactions with Matter, edited by M. Inokuti, ANL-84-28 (Argonne National Laboratory, Argonne, IL, 1984), p. 54. ${ }^{21}$ H. C. Tuckwell and Y. -K. Kim, J. Chem. Phys. 64, 333 (1976).

${ }^{22}$ K. H. Tan, C. E. Brion, Ph.E. van der Leeuw, and M. J. van der Wiel, Chem. Phys. 29, 299 (1978)

${ }^{23}$ 23. A.J. Blake and J.H. Carver, J. Chem. Phys. 47, 1038 (1967).

${ }^{24}$ C. E. Brion and F. Carnovale, Chem. Phys. 100, 291 (1985).

${ }^{25}$ M. E. Rudd and J. H. Macek, in Case Studies in Atomic Physics III, edited by E.W. McDaniel and M.R.C. McDowell (North-Holland, Amsterdam, 1972), p. 47.

${ }^{26}$ L. H. Toburen, S. T. Manson, and Y. -K. Kim, Phys. Rev. A 17, 148 (1978), 